\title{
ONE-RELATOR PRODUCTS OF TORSION-FREE GROUPS
}

\author{
by S. D. BRODSKII $\dagger$ and JAMES HOWIE
}

(Received 3 September, 1991)

If $A$ and $B$ are torsion-free groups, and $W$ is a cyclically reduced word of even length in $A * B$, it is generally conjectured that a Freiheitssatz holds, namely that each of $A$ and $B$ are embedded via the natural map into the one-relator product group $G=(A * B) / N(W)$, where $N$ denotes normal closure. If $W$ has length 2 , then $G$ is a free product of $A$ and $B$ with infinite cyclic amalgamation, and the result is obvious. The purpose of this note is to prove the Freiheitssatz in some special cases.

Definition. Let $\underline{x}=\left(x_{1}, \ldots, x_{n}\right)$ be a sequence of elements of a group $G$. We say that $x_{i}$ is isolated in $\underline{x}$ if no $x_{j}$ belongs to the cyclic subgroup generated by $x_{i}$ for $j \neq i$.

THEOREM 1. Let $A, B$ be torsion-free groups, and let $W=a_{1} b_{1} \ldots a_{k} b_{k}$ be a cyclically reduced word in $A * B$, such that some $a_{i}$ is isolated in $\left(a_{1}, \ldots, a_{k}\right)$ and some $b_{j}$ is isolated in $\left(b_{1}, \ldots, b_{k}\right)$. Let $G=(A * B) / N(W)$. Then the natural maps $A \rightarrow G$ and $B \rightarrow G$ are injective.

A second result concerns the case where one of our two groups, say $B$, is cyclic. Define the sign-index of a cyclically-reduced word

$$
W=a_{1} x^{m_{1}} \ldots a_{k} x^{m_{k}} \in A *\langle x\rangle
$$

to be the number of changes of sign in the cyclic sequence $\left(m_{1}, \ldots, m_{k}\right)$, in other words the number of negative terms in the sequence $\left(m_{1} m_{2}, \ldots, m_{k-1} m_{k}, m_{k} m_{1}\right)$. Clearly this is an even integer between 0 and $k$.

THEOREM 2. Let $A$ be a torsion-free group and let

$$
W=a_{1} x^{m_{1}} \ldots a_{k} x^{m_{k}} \in A *\langle x\rangle
$$

be a cyclically reduced word of length at least 2 and sign-index $\sigma \leq 2$. Let $G=$ $(A *\langle x\rangle) / N(W)$. Then the natural map $A \rightarrow G$ is injective. If, in addition, one of the following holds:

(i) $\sigma>0$;

(ii) $a_{1} \ldots a_{k} \in A \backslash\{1\}$

(iii) $\alpha=m_{k}+m_{1} a_{1}+m_{2}\left(a_{1} a_{2}\right)+\ldots+m_{k-1}\left(a_{1} \ldots a_{k-1}\right)$ is not a unit in $\mathbb{Q} G$;

(iv) $k \leq 3$;

then the map $\langle x\rangle \rightarrow G$ is injective, in other words $x$ has infinite order in $G$.

Remarks. (1) The first part of Theorem 2 is not new. It follows from results of Levin [7] (for the case $\sigma=0$ ) and Stallings [10] (for the case $\sigma=2$ ). The second part of the Theorem, the fact that $x$ has infinite order, is we believe, new, and equally important for the application below.

(2) It is conjectured that $\mathbb{Q} A$ has no nontrivial units for any torsion-free group $A$; in which case (iii) is satisfied whenever $k>1$, so the second part of Theorem 2 holds without the restrictions (i)-(iv). The widest class of groups for which the unit conjecture is known is that of unique product groups [8, Chapter 13] (for every pair $U, V$ of nonempty finite

† This work was supported by a visiting fellowship under SERC grant GR/F 97355.

Glasgow Math. J. 35 (1993) 99-104. 
subsets, at least one element of $U V$ is uniquely expressible as $u v$ with $u \in U$ and $v \in V$ ). In particular we see that $x$ will always have infinite order in $A$ provided $A$ is a unique-product group. Unfortunately, we do not know how to avoid the unit problem in proving Theorem 2 .

Corollary 3. Let $A, B$ be torsion-free groups, and $W=a_{1} b_{1} \ldots a_{k} b_{k}$ with $a_{i} \in A$, $b_{i} \in B, a \neq 1 \neq b$ and $k \leq 3$. Let $G=(A * B) / N(W)$. Then the natural maps $A \rightarrow G$ and $B \rightarrow G$ are injective.

Proof. Without loss of generality, we may assume that $A$ is generated by $a_{1}, \ldots, a_{k}$ and $B$ by $b_{1}, \ldots, b_{k}$. By Theorem 1 we may assume that one of the sequences $\left(a_{1}, \ldots, a_{k}\right),\left(b_{1}, \ldots, b_{k}\right)$ (say the latter) has no isolated elements. Since $k \leq 3$ it follows easily that $B$ is cyclic, say $B=\langle x\rangle$. Since $k \leq 3$ the sign-index of $W$ is either 0 or 2 , and the result follows from Theorem 2 .

Before proving the theorems, we first note an interesting consequence for the study of equations over groups. Recall that an equation $W=1$ in an unknown $X$ over a group $G$ is nonsingular if the word $W \in G *\langle X\rangle$ has nonzero exponent sum in $X$. One version of the Kervaire conjecture asserts that any nonsingular equation over any group $G$ is soluble over $G$, in other words that a solution exists in some overgroup of $G$. We say that a group $G$ is good if it satisfies this conjecture, in other words if every nonsingular equation over $G$ is soluble over $G$. Known examples of good groups include all locally residually finite groups [4] and all locally indicable groups $[2,5,9]$.

The syllable length of the equation $W=1$ is defined to be the length of $W$ as a (cyclically reduced) word in the free product $G *\langle X\rangle$. In particular, this length is always assumed to be even. A result of Edjvet [3] asserts that any equation of the form $a X^{m} b X^{n}=1$ with $m \neq-n$ has a solution over any group $G$, except possibly if $a$ has order 2 in $G$ and $b$ has order 3 , or vice versa. In particular, any equation of syllable length 4 over any torsion-free group $G$ has a solution over $G$.

CoRollary 4. If $G$ is a good, torsion-free group, then any equation over $G$ of syllable length at most 14 has a solution over $G$.

Proof. The result follows from the definition of good unless the equation is singular. In this case the group $H=(G * \mathbb{Z}) / N(W)$ is an $H N N$-extension of a group $A=\left(G_{1} * \ldots *\right.$ $\left.G_{k}\right) / N(V)$, where $k \geq 2$, each $G_{i} \cong G$, and $V$ is a word of length at most 7 in the free product, that contains letters from both $G_{1}$ and $G_{k}$. (Here the associated subgroups are $G_{1} * \ldots * G_{k-1}$ and $G_{2} * \ldots * G_{k}$, which are naturally embedded in $A$ by Corollary 3 .)

Remark. The proofs of the results use the weight test method of Bogley and Pride [1]. Recall [1] that a weight function for a relative presentation

$$
\left\langle G, X_{1}, \ldots, X_{m} \mid W_{1}, \ldots, W_{n}\right\rangle
$$

of a group $H$ (where $G$ is a group and the $W_{i}$ are words in $G *\left\langle X_{1}, \ldots, X_{m}\right\rangle$ ) is a real valued function $w$ on the edges of the star graph of the presentation. A weight function is aspherical if it is non-negative; $w\left(g_{1}\right)+\ldots+w\left(g_{t}\right) \leq(t-2) \pi$ if $W_{i}=g_{1} X_{\alpha(1)}^{ \pm 1} \ldots g_{t} X_{\alpha(t)}^{ \pm 1}$ for some $i$; and $w(R) \geq 2 \pi$ whenever $R$ is a cyclically reduced closed path in the star graph labelled by a relation in $G$ (among the coefficients $g_{i}$ ). Here $w(R)$ denotes the sum of the weights of the edges in $R$. A relative presentation with an aspherical weight 
function is called aspherical, and for such a presentation $G$ always embeds in $H$ via the natural map. (For details, see [1].)

If $w$ is an aspherical weight function with the additional property that $w(R) \geq \pi$ for any reduced non-closed path $R$ in the star graph labelled by a relation among the $g_{i}$, then the methods of [1] can also be used to show that no nonempty, cyclically reduced word in the $X_{i}$ represents the identity element of $H$, in other words the $X_{i}$ form a basis for a free subgroup of $H$. We omit the details.

Proof of Theorem 1. Without loss of generality, we may assume that $A$ is generated by $a_{1}, \ldots, a_{k}$, and $B$ by $b_{1}, \ldots, b_{k}$; and that $a_{i}$ is isolated in $\left(a_{1}, \ldots, a_{k}\right)$ and $b_{j}$ in $\left(b_{1}, \ldots, b_{k}\right)$. The theorem certainly holds if the equation

$$
a_{1} X b_{1} X^{-1} \ldots a_{k} X b_{k} X^{-1}=1
$$

is soluble over $A * B$. The proof proceeds by applying the weight test method to this equation, or equivalently to the relative presentation

$$
\left\langle A * B, X \mid a_{1} X b_{1} X^{-1} \ldots a_{k} X b_{k} X^{-1}\right\rangle .
$$

The star graph of this relative presentation consists of two disjoint bouquets of circles, with $k$ circles in each. One of these corresponds to the $A$-letters, and the other to the $B$-letters. Define a weight function $w$ by $w\left(a_{i}\right)=w\left(b_{j}\right)=0, w\left(a_{m}\right)=\pi$ for $m \neq i$, and $w\left(b_{m}\right)=\pi$ for $m \neq j$. Then certainly $w$ is non-negative, and the sum of the weights is $(2 k-2) \pi$. To see that $w$ is aspherical, it remains only to check that no nonempty closed path of weight less than $2 \pi$ in the star graph represents the identity element of $A$ or of $B$.

Any such path has the form $a_{i}^{t}$ or $b_{j}^{t}$ for some $t \neq 0$; or $a_{m}^{ \pm 1} a_{i}^{t}$ for some $t \in \mathbb{Z}, m \neq i$ or $b_{m}^{ \pm 1} b_{j}^{t}$ for some $t \in \mathbb{Z}, m \neq j$ (up to cyclic permutation). The first two possibilities are ruled out by the condition that $A$ and $B$ are torsion-free (for example, if $a_{i}^{\prime}=1$ then $a_{i}=1$, contradicting the hypothesis that $W$ is cyclically reduced). The remaining possibilities are ruled out by the hypothesis that $a_{i}$ and $b_{j}$ are isolated.

Hence $w$ is aspherical, and so the natural maps $A \rightarrow G, B \rightarrow G$ are injective, as claimed.

Proof of Theorem 2. Suppose first that $W$ has sign-index $\sigma=2$ (condition (i) of the theorem). Then the star graph of the relative presentation

$$
\langle A, x \mid W\rangle
$$

consists of two vertices, $x$ and $x^{-1}$, a loop at each, and a collection (possibly empty) of edges joining $x^{-1}$ to $x$. Let $w$ be the weight function that assigns the weight 0 to each of the two loops, and $\pi$ to each of the other edges. Then the only closed paths of weight less than $2 \pi$ are powers of the loops, which cannot be relations in $A$ since $A$ is torsion-free. Hence this is an aspherical weight function for (2), and (2) is aspherical. In particular, $A$ is naturally embedded in $G$. Moreover, any path in the star graph from $x^{-1}$ to $x$ has weight at least $\pi$, so $x$ has infinite order in $G$.

Next suppose that $W$ has sign-index $\sigma=0$. In other words, after replacing $x$ by $x^{-1}$ if necessary, $W$ is a positive word in $x$. Then certainly $A$ is embedded in $G$, by Levin's Theorem [7]. Indeed, Levin shows that $G$ has a homomorphic image isomorphic to the wreath product of $A$ by a cyclic group of order $n$, where $n=m_{1}+\ldots+m_{k}$. The cyclic 
group of order $n$ is generated by an element $t$, conjugation by $t$ permutes the $n$ copies of $A$ in the direct product, and $x$ maps to the element

$$
\left(a_{1}^{-1}, 1, \ldots, 1, a_{2}^{-1}, 1, \ldots, 1, a_{k}^{-1}, 1, \ldots, 1\right) t
$$

(Here $a_{1}^{-1}$ is separated from $a_{2}^{-1}$ by $\left(m_{1}-1\right) 1$ 's, and so on, so that each $a_{p}^{-1}$ occurs in the $\left(m_{1}+\ldots+m_{p-1}+1\right)^{\prime}$ th place in the vector.)

Hence $x^{n}$ maps to an element of the direct power of $A$, each component of which is conjugate to the inverse of the product (in order) of the $a_{i}$. Since $A$ is torsion-free, it follows that $x$ has infinite order in $G$, except possibly if this product $a_{1} \ldots a_{k}$ is the identity in $A$, in other words if condition (ii) fails. From now on we assume that this is the case.

The injection $A \rightarrow G$ is then split, via the map $G \rightarrow A$ that is the identity on $A$ and sends $x$ to 1 . The kernel of the splitting map has a presentation

$$
K=\left\langle x_{a}(a \in A) \mid x_{a a_{1}}^{m_{1}} x_{a a_{1} a_{2}}^{m_{2}} \ldots x_{a}^{m_{k}}(a \in A)\right\rangle,
$$

by Reidemeister-Schreier rewriting, where $x_{a}=a x a^{-1}$. If $x$ has finite order in $G$, then $K$ is generated by elements of finite order, so

$$
H_{1}(K ; \mathbb{Q}) \cong K^{a b} \otimes_{\mathbb{Z}} \mathbb{Q}=0
$$

But $A$ acts regularly on the generators and relations of $(3)$, and so $H_{1}(K ; \mathbb{Q})$ can be computed explicitly as the cokernel of the Fox derivative $\partial: \mathbb{Q} A \rightarrow \mathbb{Q} A$ of $W$ with respect to $x$. Since $H_{1}(K ; \mathbb{Q})=0, \partial$ is surjective (and hence an isomorphism). But $\partial$ is given by right multiplication by $\alpha$ on $\mathbb{Q} A$, so $\alpha$ must be a unit in $\mathbb{Q} A$. Hence condition (iii) fails.

Suppose, finally, that $k \leq 3$. If $k=1$ then (ii) holds, so $x$ has infinite order in $G$. If $A$ is cyclic, then $\mathbb{Q} A$ has no nontrivial units, in particular no units of the form $\alpha$ with $k>1$, so condition (iii) holds. In particular this is the case if $k=2$, for then $A=\left\langle a_{1}, a_{2}\right\rangle$ with $a_{1} a_{2}=1$.

Thus we are reduced to the case where $k=3, A=\left\langle a_{1}, a_{2}\right\rangle$ is non-cyclic, and $W=a_{1} x^{p} a_{2} x^{q}\left(a_{1} a_{2}\right)^{-1} x^{r}$ for some $p, q, r \geq 1$. The star graph of (2) consists of $p+q+r$ edges, all joining the vertex $x^{-1}$ to $x$. Three of these are labelled $a_{1}, a_{2}, a_{3}$, and the others are labelled by the identity of $A$. Let $w$ be the weight function that assigns a weight of $\pi / 3$ to each of the edges $a_{1}, a_{2}, a_{3}$, and $\pi$ to each of the remaining edges, The only non-closed, reduced paths of weight less than $\pi$ are labelled $a_{i}^{ \pm 1}$ for $i=1,2,3$, none of which is a relation in $A$. Similarly, the only closed, cyclically reduced paths of weight less than $2 \pi$ are labelled (up to cyclic permutation and inversion) $a_{i}, a_{i} a_{j}^{-1}$ for $i \neq j$, or $a_{i} a_{j}^{-1} a_{i} a_{k}^{-1}$ for $j \neq i \neq k$. Given that $A$ is torsion-free, non-cyclic, and generated by $a_{1}, a_{2}, a_{3}$ with $a_{1} a_{2} a_{3}=1$, it can readily be verified that only the last of these, with $j \neq k$, are possible relations in $A$. If no such word is a relation in $A$ then (2) is aspherical. Hence $x$ has infinite order in $G$, and we are done.

Suppose then that a word $a_{i} a_{j}^{-1} a_{i} a_{k}^{-1}$ is a relation in $A$, with $i, j, k$ pairwise distinct. Without loss of generality we may assume that $i=1, j=2$ and $k=3$. Then $A$ is a homomorphic image of

$$
\hat{A}=\left\langle a_{1}, a_{2}, a_{3} \mid a_{1} a_{2} a_{3}, a_{1} a_{2}^{-1} a_{1} a_{3}^{-1}\right\rangle \cong\left\langle x, y \mid x y x^{-1} y^{2}\right\rangle .
$$


But no proper homomorphic image of $\hat{A}$ is torsion free and noncyclic, so $A \cong \hat{A}$. Now in this case there is an alternative weight function: take $w\left(a_{2}\right)=w\left(a_{3}\right)=0$, and $w(e)=\pi$ for all other edges. This makes (2) aspherical (compare [1, Theorem 3.2, Case 3], and also has the property that no reduced, nonclosed path of weight less than $\pi$ is labelled by a relation in $A$. Once again, $x$ has infinite order in G. This completes the proof.

Alternatively, we can complete the proof using the fact that the group $\hat{A}$ is a torsion-free one-relator group, hence locally indicable [2], and so $\mathbb{Q} \hat{A}$ has no nontrivial units (see for example [8]). Now apply (iii).

Concluding remarks. 1. Our method of proof also shows that the one-relator product group $G=(A * B) / N(W)$ in Corollary 3 is itself torsion-free, except in the case where $W$ is a proper power in $A * B$. Indeed, we showed that either $G$ is (essentially) a one-relator group, or $G$ is (a subgroup of) a group given by an aspherical relative presentation over a torsion-free group. In either case, $G$ can have torsion only if the relator is a proper power. (See [1] for the relative presentation case).

2. It is reasonable to expect that the length restrictions on Corollaries 3 and 4 could be relaxed somewhat. More subtle methods of proof would be required however, as one can construct examples of length 8 words in $A * B$ such that neither factor group is cyclic, yet no aspherical weight function exists. One such example is $W=a b a d c b c d^{-1}$, where $A=\langle a, c\rangle$ and $B=\langle b, d\rangle$ are both free of rank 2 . One possible approach might be to use the cycle test of Huck and Rosebrock [6] in place of the weight test.

3. Finally, the intrusion of the unit problem into Theorem 2 raises the question of whether it can be avoided. Explicitly, one can ask, given any positive word $W=$ $a_{1} x \ldots a_{k} x \in A *\langle x\rangle$, with at least one of the $a_{i}$ nontrivial in $A$, whether $x$ has infinite order in the one-relator product $G=(A *\langle x\rangle) / N(W)$. Theorem 2 provides some evidence for an affirmative answer, but not yet a complete proof. It is rather curious that the infinite order part of Theorem 2 should be so much easier for words of sign-index 2 than for positive words.

ACKNowledgements. We are grateful to the referee for useful comments, in particular for pointing out and filling a gap in our original proof of Theorem 2 . We are also grateful to Steve Pride for a number of helpful remarks.

\section{REFERENCES}

1. W. A. Bogley and S. J. Pride, Aspherical relative presentations, Proc. Edinburgh Math. Soc. 35 (1992), 1-34.

2. S. D. Brodskĩ, Equations over groups and groups with a single defining relator, Siberian Math. J. 25 (1984), 235-251.

3. M. Edjvet, Equations over groups and a theorem of Higman, Neumann and Neumann, Proc. London Math. Soc. 62 (1991), 563-589.

4. M. Gerstenhaber and O. S. Rothaus, The solution of sets of equations in groups, Proc. Nat. Acad. Sci. USA 48 (1962), 1531-1533.

5. J. Howie, On pairs of 2-complexes and systems of equations over groups, J. reine angew. Math. 324 (1981), 165-174.

6. G. Huck and S. Rosebrock, Hyperbolische Teste auf Diagrammatische Reduzierbarkeit für Standard 2-Komplexe, Preprint (1990). 
7. F. Levin, Solutions of equations over groups, Bull. Amer. Math. Soc. 68 (1962), 603-604.

8. D. Passman, The algebraic structure of group rings, (Wiley, 1977).

9. H. Short, Topological methods in group theory: the adjunction problem, Ph.D. thesis, University of Warwick (1984).

10. J. R. Stallings, A graph-theoretic lemma and group embeddings, Annals of Mathematics Studies 111 (1987), 145-155.

Department of Mathematics

Afula Research Institute

AfUlA, IsraEL
Department OF Mathematics HERIOT-WATT UNIVERSITY RICCARTON

EDINBURGH, EH14 4AS 